\title{
Voltage-biased quantum wire with impurities
}

\author{
Reinhold Egger and Hermann Grabert \\ Fakultät für Physik, Albert-Ludwigs-Universität, Hermann-Herder-Straße 3, D-79104 Freiburg, Germany
}

\begin{abstract}
The bosonization technique to describe correlated electrons in a one-dimensional quantum wire containing impurities is extended to include an applied voltage source. The external reservoirs are shown to lead to a boundary condition for the boson phase fields. We use the formalism to investigate the channel conductance, electroneutrality, and charging effects.
\end{abstract}

PACS numbers: 72.10.-d, 73.40.Gk

The puzzling physical properties of one-dimensional correlated fermions at low temperatures can most conveniently be described within the bosonization technique. This method [1] 3] allows for an exact treatment of Coulomb interactions that hamper most other theoretical approaches. In this Letter, we describe how a voltage bias can properly be incorporated in terms of a boundary condition for the bosonized phase fields. The formalism is similar in spirit to Landauer's approach developed for noninteracting electrons [4]. The capacity of our concept is demonstrated for a single impurity embedded into the correlated one-dimensional (1D) electron liquid, a problem that has attracted considerable theoretical interest [5,6], and is beginning to find experimental realizations [7].

In order to describe Coulomb interactions adequately, one has to specify the setup under consideration. If one deals with a 1D channel in heterostructures, a "quantum wire" [8], the interactions are usually screened due to the presence of metallic gates near the channel. This leads to a Luttinger liquid [3] characterized by an interaction constant $g$ (we only discuss the spinless single-channel case in the following). The noninteracting case corresponds to $g=1$, and the presence of (repulsive) Coulomb interactions implies $g<1$. Quantities of principal interest are the channel conductance in the presence of interactions and the capacitance $C=Q / U$ of a junction or impurity ( $U$ is the two-terminal voltage, and $Q$ denotes the charge on the junction). We present an approach that allows one to address these problems for finite voltage at arbitrary interaction strength and junction transmittance.

Our treatment is based on the standard bosonization approach [1 3], which is applicable in the low-energy regime where only excitations near the Fermi surface are relevant. The electron creation operator can be expressed in terms of boson phase fields $\theta(x)$ and $\phi(x)$,

$\psi^{\dagger}(x)=\sqrt{\frac{\omega_{c}}{2 \pi v_{F}}} \sum_{p= \pm} \exp \left[i p k_{F} x+i \sqrt{\pi}[p \theta(x)+\phi(x)]\right]$,

where $\omega_{c}=v_{F} k_{F}$ is the proper bandwidth cutoff for the linearized dispersion relation employed in the bosonization (we put $\hbar=1$ ). The phase fields obey the equal-time commutation relations

$$
\left[\phi(x), \theta\left(x^{\prime}\right)\right]=-(i / 2) \operatorname{sgn}\left(x-x^{\prime}\right)
$$

such that the canonical momentum for the $\theta$ field is $\Pi=$ $\partial_{x} \phi$. The boson representation for the electron density operator is then given by

$$
\rho(x)=\frac{k_{F}}{\pi}+\frac{1}{\sqrt{\pi}} \partial_{x} \theta(x)+\frac{k_{F}}{\pi} \cos \left[2 k_{F} x+2 \sqrt{\pi} \theta(x)\right] .
$$

The first term is the background charge, the second term stands for the sum of right- and left-moving densities $\rho_{ \pm}$, and the last term describes interference between rightand left-movers [2]. The clean Luttinger liquid is described by the Euclidean action [3]

$$
S_{0}=\frac{v_{F}}{2} \int d x d \tau\left[\frac{1}{v_{F}^{2}}\left(\partial_{\tau} \theta\right)^{2}+\frac{1}{g^{2}}\left(\partial_{x} \theta\right)^{2}\right],
$$

where $v_{F}$ is the Fermi velocity and $g \leq 1$ the interaction constant. A short-ranged impurity at $x=0$ results in the generic contribution [5]

$$
S_{I}=V \int d \tau \cos [2 \sqrt{\pi} \theta(0, \tau)]
$$

where the dimensionless impurity strength $\lambda=\pi V / \omega_{c}$ tunes the junction resistance.

Now let us consider a $1 \mathrm{D}$ quantum wire coupled to external reservoirs, see Fig. 1. The coupling of the $1 \mathrm{D}$ channel to the $2 \mathrm{D}$ or $3 \mathrm{D}$ reservoirs is assumed to occur by adiabatic widening of the channel. This models ideal reservoirs in the sense of Landauer 4 . The reservoirs are macroscopic, totally incoherent devices kept at fixed chemical potential, and there are no reflections of particles entering the reservoirs. The left (right) reservoir at $x \rightarrow-\infty(x \rightarrow \infty)$ has chemical potential $\mu_{-\infty}\left(\mu_{\infty}\right)$, and the difference in chemical potentials is the two-terminal voltage $U=$ $\left(\mu_{-\infty}-\mu_{\infty}\right) / e$. The mean chemical potential $\left(\mu_{-\infty}+\right.$ $\left.\mu_{\infty}\right) / 2$ corresponds to the background charge $k_{F} / \pi$. 


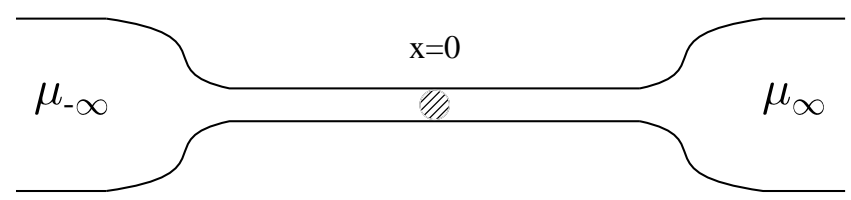

FIG. 1. General setup studied in this work: A 1D quantum wire is coupled to 3D reservoirs, which are held at chemical potentials $\mu_{-\infty}$ and $\mu_{\infty}$. The left reservoir injects right-movers at the stationary excess density $\left\langle\rho_{+}\right\rangle$, and the right one injects left-movers with density $\left\langle\rho_{-}\right\rangle$. The striped area stands for a scatterer at $x=0$.

Applying a voltage means that one has a non-equilibrium influx of currents from the reservoirs: The reservoir at $x \rightarrow-\infty$ injects right-movers at some stationary excess density, and similarly the density of left-movers at $x \rightarrow \infty$ is diminished,

$$
\left\langle\rho_{ \pm}(x)\right\rangle= \pm \frac{e U}{4 \pi v_{F}} \quad \text { as } x \rightarrow \mp \infty .
$$

While in a clean system the densities of right- and leftmovers imposed by the reservoirs spread homogeneously along the wire, in the presence of scatterers one does not know beforehand what the densities of right- (left-) movers at $x \rightarrow \infty(x \rightarrow-\infty)$ are. These densities follow from our theory.

The boundary condition (41), formulated in terms of the average densities of right- or left-moving fermions in the quantum wire as the reservoirs are approached, can equivalently be expressed in terms of the $\theta$ field by noting that

$$
\rho_{+}+\rho_{-}=\frac{1}{\sqrt{\pi}} \partial_{x} \theta, \quad \rho_{+}-\rho_{-}=\frac{i}{v_{F} \sqrt{\pi}} \partial_{\tau} \theta .
$$

The boundary condition for the boson phase field is therefore

$$
\left( \pm \frac{\partial}{\partial x}+\frac{i}{v_{F}} \frac{\partial}{\partial \tau}\right)\langle\theta(x \rightarrow \mp \infty, \tau=0)\rangle=\frac{e U}{2 \sqrt{\pi} v_{F}},
$$

where the time $\tau=0$ has been picked by convention.

To study the inhomogeneous quantum wire in presence of an external voltage, we consider the generating functional $Z(y, \mu)=\langle\exp [2 \sqrt{\pi} i \mu \theta(y)]\rangle$. We formally solve for $Z$ by introducing an auxiliary field $q(\tau)=2 \sqrt{\pi} \theta(x=$ $0, \tau)$, with the constraint enforced by a Lagrange multiplier field $\Lambda(\tau)$. Then one has the effective action

$$
S_{e}[\theta, \Lambda, q]=\frac{v_{F}}{2} \int d x d \tau\left[\frac{1}{v_{F}^{2}}\left(\partial_{\tau} \theta\right)^{2}+\frac{1}{g^{2}}\left(\partial_{x} \theta\right)^{2}\right]
$$

$$
\begin{aligned}
& +V \int d \tau \cos q(\tau)-2 \sqrt{\pi} i \mu \theta(y, 0) \\
& +i \int d \tau \Lambda(\tau)[2 \sqrt{\pi} \theta(0, \tau)-q(\tau)]
\end{aligned}
$$

The $\theta$ part of this effective action is Gaussian and can therefore be treated exactly by solving the classical EulerLagrange equations. One can always decompose the solution $\theta$ into an homogeneous part $\theta_{h}$ for the equilibrium system $(U=0)$, and a particular solution $\theta_{p}$ subject to the boundary condition (5). At the same time, we decompose the field $\Lambda=\Lambda_{h}+\Lambda_{p}$, such that

$$
\frac{1}{v_{F}^{2}} \frac{\partial^{2} \theta_{p}}{\partial \tau^{2}}+\frac{1}{g^{2}} \frac{\partial^{2} \theta_{p}}{\partial x^{2}}=\frac{2 \sqrt{\pi} i}{v_{F}} \delta(x) \Lambda_{p}(\tau) .
$$

The most general solution permitted by Eq. (7) which fulfills Eq. (5) requires a $\tau$ independent $\Lambda_{p}$ and takes the form

$$
\theta_{p}(x, \tau)=\frac{q_{0}}{2 \sqrt{\pi}}-\frac{e \varphi}{2 \sqrt{\pi} v_{F}}|x|-i \tau \frac{e(U-\varphi)}{2 \sqrt{\pi}} .
$$

The quantity $\varphi$ is related to the zero mode of the Lagrange multiplier field, $\Lambda_{p}=i e \varphi / 2 \pi g^{2}$.

With the boson propagators

$$
F(x, \omega)=\frac{\pi g}{|\omega|} \exp \left(-\left|g \omega x / v_{F}\right|\right)
$$

the homogeneous part can be written in terms of the Fourier components $\Lambda_{h}(\omega)$,

$\theta_{h}(x, \tau)=\frac{-i}{\sqrt{\pi}} \int \frac{d \omega}{2 \pi} e^{i \omega \tau}\left[\Lambda_{h}(\omega) F(x, \omega)-\mu F(x-y, \omega)\right]$.

Inserting $\theta_{h}+\theta_{p}$ into Eq. (6), the action becomes Gaussian in $\Lambda_{h}$, which can therefore easily be integrated out. Adding the particular solution (8) also onto $q(\tau)$ in view of $q(\tau)=2 \sqrt{\pi} \theta(0, \tau)$, one obtains for the generating functional

$$
\begin{aligned}
& \langle\exp [2 \sqrt{\pi} i \mu \theta(x)]\rangle=W(x)^{\mu^{2}}\left\langle e^{-i \mu e \varphi|x| / v_{F}}\right. \\
& \left.\times \exp \left[i \mu\left(q_{0}+\int \frac{d \omega}{2 \pi} q(\omega) \frac{F(x, \omega)}{F(0, \omega)}\right)\right]\right\rangle,
\end{aligned}
$$

where the average over the zero modes $q_{0}$ and $\varphi$ of the auxiliary fields and over the $q$ fluctuations $q(\tau)=$ $(2 \pi)^{-1} \int d \omega q(\omega) \exp (i \omega \tau)$ has to be taken using the action

$$
\begin{aligned}
S & =\int \frac{d \omega}{2 \pi} \frac{q(\omega) q(-\omega)}{4 F(0, \omega)}+\frac{e \varphi}{2 \pi g^{2}} \int d \tau q(\tau) \\
& +V \int d \tau \cos \left[q_{0}-i e(U-\varphi) \tau+q(\tau)\right] .
\end{aligned}
$$

The function $W(x)=(1+|x| / \alpha)^{-g}$ with the microscopic lengthscale $\alpha=v_{F} / 2 g \omega_{c}$ does not depend on impurity properties. 
The effect of the external voltage can now be read off from Eqs. (9) and (10). First, the average density [the first two terms in Eq. (11)] is in general discontinuous at the impurity location due to particle reflection,

$$
\bar{\rho}=\frac{k_{F}}{\pi}-\frac{e \varphi}{2 \pi v_{F}} \operatorname{sgn}(x) .
$$

For the noninteracting case, $g=1$, one can show from the exact solution of the equivalent Schrödinger equation that $\varphi$ is the usual four-terminal voltage measured near the barrier [9]. In that case, $\varphi=R U$, where $R=1 /(1+$ $\left.\lambda^{-2}\right)$ is the reflection coefficient of the barrier.

Second, in the absence of a scatterer, $\lambda=0$, one always finds $\varphi=0$ (see below), and the right- and left-moving densities are spatially homogeneous along the wire. Since they are determined by Eq. (4), the current is

$$
I=\frac{i}{\sqrt{\pi}}\left\langle\frac{\partial \theta}{\partial \tau}\right\rangle=e v_{F}\left(\left\langle\rho_{+}\right\rangle-\left\langle\rho_{-}\right\rangle\right)=\frac{e^{2}}{h} U
$$

This yields the perfect two-terminal conductance $e^{2} / h$ in agreement with recent theoretical work [10] and an experimental study of a quantum wire [7]. The conductance $g e^{2} / h$ discussed in Ref. [5] is not the two-terminal conductance but a low-frequency microwave conductance.

To describe coupling to an external voltage, previous studies have often added a term to the Hamiltonian of the form 5.11]

$$
\tilde{H}=e \tilde{\varphi} \theta(0) / \sqrt{\pi}=\frac{e \tilde{\varphi}}{2 \pi} q,
$$

where $\tilde{\varphi}$ is the "voltage drop" [12]. From Eq. (10), if one tentatively identifies $\varphi$ with $\tilde{\varphi}$, one observes that Eq. (12) should be modified by a factor $1 / g^{2}$. This factor can be understood in terms of the interaction energy of density fluctuations with the nonequilibrium background charge (11) deviating from $k_{F} / \pi$,

$$
\begin{aligned}
& \int d x \int d x^{\prime} \frac{1}{\sqrt{\pi}} \partial_{x} \theta(x) U_{c}\left(x-x^{\prime}\right)\left(\frac{-e \varphi \operatorname{sgn}\left(x^{\prime}\right)}{2 \pi v_{F}}\right) \\
& =-\frac{e \varphi}{2 \sqrt{\pi}}\left(\frac{1}{g^{2}}-1\right) \int d x \operatorname{sgn}(x) \partial_{x} \theta(x),
\end{aligned}
$$

where $U_{c}\left(x-x^{\prime}\right)$ is the screened Coulomb interaction, and the last line holds for a Luttinger liquid. Furthermore, it should be noted that in Eq. (10) the cosine part due to the impurity has acquired a term linear in time, which is reminiscent of the Josephson relations. This shows that the external voltage cannot be fully incorporated by simply adding a term like Eq. (12) to the Hamiltonian. In general, it is necessary to treat external reservoirs via boundary conditions [13].

From the above considerations, we see that the action (10) describes a voltage-biased 1D quantum wire containing a scatterer for the entire range of parameters. When evaluating Eq. (9), we still have to average over the zero mode of the Lagrange multiplier field. Hence $\varphi$ is generally a fluctuating quantity. In the two limiting cases of transmission zero and one, the fluctuations do vanish. In the latter case, $\lambda=0$, the $q$ average is Gaussian, and one finds $\varphi=0$ due to the infrared singularity of the first term in the action [5]. On the other hand, for $\lambda \rightarrow \infty$, the cosine term in Eq. (10) strictly enforces $\varphi=U$. In the following, we will only consider the two fixed-point values $\varphi=0$ and $U$ corresponding to a very small and a very high barrier, respectively. Near these limiting cases, further analytic progress can be made.

We start by calculating the nonequilibrium electron density. Equivalent to an explicit real-time calculation, we first analytically continue to imaginary values of $U$ and $\varphi$, and after performing the $q$ average, we rotate back to real values of $U$ and $\varphi$. Let us first study the case of a very weak scatterer, such that we can put $\varphi=0$ and then use perturbation theory in the impurity strength. The antisymmetric charge distribution

$$
q(x)=-e[\langle\rho(x)\rangle-\langle\rho(-x)\rangle] / 2 \quad(x>0)
$$

can be computed by expressing the density operator (11) in terms of the generating functional (9). Lowest-order perturbation theory in $\lambda$ yields

$$
\begin{aligned}
q(x) & =\frac{e \lambda k_{F}}{\pi} \sin \left(2 k_{F} x\right) \frac{\sqrt{\pi}}{2 \Gamma(g)}(x / \alpha)^{-(g-1 / 2)} \\
& \times\left(e U / \omega_{c}\right)^{g-1 / 2} J_{g-1 / 2}\left(g e U x / v_{F}\right)
\end{aligned}
$$

where $J_{\nu}(x)$ is a Bessel function of the first kind [14]. Near the impurity, for $x \ll\left(e U / v_{F}\right)^{-1}$, from properties of the Bessel function,

$$
q(x)=\frac{e \lambda k_{F}}{2 \sqrt{\pi} \Gamma(g) \Gamma(g+1 / 2)} \sin \left(2 k_{F} x\right)\left(e U / 2 \omega_{c}\right)^{2 g-1} .
$$

The asymmetric charge mode $q(x)$ is $2 k_{F}$-periodic but decays only on the lengthscale $v_{F} / e U$. There is no localized charge sitting on the impurity. However, the $x$ integration over (13) gives a finite total charge, and hence a finite nonlinear capacitance $C=Q / U$,

$$
C(U)=\frac{e^{2} \lambda / \omega_{c}}{8 \sqrt{\pi} \Gamma(g) \Gamma(g+1 / 2)}\left(\frac{e U}{2 \omega_{c}}\right)^{2 g-2} .
$$

This lowest-order perturbational result in the weakscattering regime breaks down for small voltages, $e U / 2 \omega_{c} \ll \lambda^{1 /(2-2 g)}$.

In the opposite case of a strong scatterer, $\lambda \gg 1$, we can put $\varphi=U$. From Eq. (9), the $2 k_{F}$-part of the asymmetric charge mode takes the form

$$
q_{2 k_{F}}(x)=-\frac{e k_{F}}{\pi} \cos \left(2 k_{F} x\right) \sin \left(e U x / v_{F}\right)(x / \alpha)^{-g},
$$

which implies a finite total charge $Q_{2 k_{F}}$. This charge turns out to be linear in $U$, and therefore one has a finite $U=0$ capacitance 


$$
C_{2 k_{F}}=\frac{e^{2}}{4 \pi \omega_{c}} g^{-g} \Gamma(2-g) \sin \left[\frac{\pi}{2}(1-g)\right] .
$$

However, $q_{2 k_{F}}(x)$ does not include the constant term $\bar{q}=$ $e U / 2 \pi v_{F}$ coming from the change in background density (11). That term leads to a charge $Q_{0}=L e^{2} U / 4 \pi v_{F}$, which diverges with the system length $L$. The applied voltage polarizes the capacitance between the wire and the metallic screening gate. This large shunt capacitance renders the observation of charging effects, i.e. of $C_{2 k_{F}}$, impossible for a single scatterer. A similar situation is encountered for a single tunnel junction coupled to metallic leads, where charging effects are normally absent 15.

For an island formed by two strong impurities, the $2 k_{F}$ capacitance is observable since the capacitance of the island is not affected by the shunt capacitance between the remaining wire and the gate. Taking two impurities at $x= \pm R / 2$ and applying our boundary condition (5), we now have two zero modes from the respective Lagrange multipliers. One $(\varphi)$ corresponds to the four-terminal voltage found in the single-impurity case, and the other $\left(\varphi_{G}\right)$ corresponds to a gate voltage applied to the island $-R / 2<x<R / 2$. While again analytic results are not available for the entire range of parameters, it is possible to calculate the total charge $Q_{I}$ sitting on the island in the limit of large barriers and for $k_{F} R \gg 1$. We obtain

$$
\begin{aligned}
Q_{I} & =\frac{e R}{\pi}\left(k_{F}+e \varphi_{G} / 2 v_{F}\right)+2 C_{2 k_{F}} \varphi_{G} \\
& -e \Gamma(1-g) \sin [\pi(1-g) / 2] / \pi g^{g},
\end{aligned}
$$

where $C_{2 k_{F}}$ is given in Eq. (15). The first term $\sim R$ arises due to the slow component of the density operator (11), while the remaining two terms come from the $2 k_{F}$ component. The resonant tunneling condition can then be derived by noting that $Q_{I} / e$ is confined to integer values in the large-barrier limit under consideration here. Hence, the spacing of the resonances as a function of $\varphi_{G}$ is found to be

$$
e \Delta \varphi_{G}=\left(R / 2 \pi v_{F}+2 C_{2 k_{F}} / e^{2}\right)^{-1} .
$$

Therefore $C_{2 k_{F}}$ leads to an experimentally measurable resonance shift compared to previous results $[5$ which neglected charging effects in interacting $1 \mathrm{D}$ metals.

Let us finally comment on the issue of electroneutrality in a Luttinger liquid. The spatial change in the background charge density (11) induces an influence charge density of opposite sign on the metallic gate, such that overall electroneutrality is maintained. However, the Luttinger liquid interaction is not able to enforce electroneutrality within the $1 \mathrm{D}$ quantum wire alone. In the complete absence of a gate, since there is no internal screening within the wire, the Coulomb potential becomes long-ranged, $U_{c}\left(x-x^{\prime}\right) \sim\left|x-x^{\prime}\right|^{-1}$. From the Euler-Lagrange equation corresponding to Eq. (7) and the boundary condition (5), one finds immediately that then $\varphi=0$. As a consequence, for a long-ranged Coulomb potential, electroneutrality is maintained automatically within the wire.

In conclusion, the theoretical description of a voltagebiased 1D quantum wire (Luttinger liquid) containing elastic potential scatterers has been given. Our boundary condition method can easily be adopted to the case of more than one channel (e.g., the spin- $\frac{1}{2}$ case), and it can straightforwardly be generalized to a real-time and finitetemperature formalism.

[1] A. Luther and I. Peschel, Phys. Rev. B 9, 2911 (1974); Phys. Rev. Lett. 32, 992 (1974).

[2] V.J. Emery, in Highly Conducting One-Dimensional Solids, ed. by J.T. Devreese (Plenum, New York, 1979).

[3] F.D.M. Haldane, J. Phys. C 14, 2585 (1981); Phys. Rev. Lett. 47, 1840 (1981).

[4] R. Landauer, IBM J. Res. Dev. 1, 223 (1957); Phil. Mag. 21, 863 (1970); Z. Phys. B 68, 217 (1987).

[5] C.L. Kane and M.P.A. Fisher, Phys. Rev. Lett. 68, 1220 (1992); Phys. Rev. B 46, 15233 (1992).

[6] K.A. Matveev, D. Yue, and L.I. Glazman, Phys. Rev. Lett. 71, 3351 (1993); A. Furusaki and N. Nagaosa, Phys. Rev. B 47, 4631 (1993); M. Fabrizio and A.O. Gogolin, ibid. 51, 17827 (1995); P. Fendley, A.W.W. Ludwig, and H. Saleur, Phys. Rev. Lett. 74, 3005 (1995); R. Egger and H. Grabert, ibid. 75, 3505 (1995).

[7] S. Tarucha, T. Honda, and T. Saku, Solid State Comm. 94, 413 (1995).

[8] A.O. Gogolin, Ann. Phys. Fr. 19, 411 (1994); G. Timp, in Mesoscopic Phenomena in Solids, ed. by B.L. Al'tshuler et al. (Elsevier, Amsterdam, 1990).

[9] M. Büttiker, Phys. Rev. Lett. 57, 1761 (1986).

[10] D.L. Maslov and M. Stone, Phys. Rev. B 52, R5539 (1995); V.V. Ponomarenko, ibid. 52, R8666 (1995); I. Safi and H.J. Schulz, ibid. 52, R17040 (1995).

[11] M. Sassetti and U. Weiss, Europhys. Lett. 27, 311 (1994).

[12] The expression (12) follows from $\tilde{H}=\int d x \mu(x) \rho(x)=$ $\int d x(-e \tilde{\varphi} \operatorname{sgn}(x) / 2) \partial_{x} \theta(x) / \sqrt{\pi}$, which tacitly assumes that the electro-chemical potential $\mu(x)$ is independent of $\rho(x)$.

[13] We note that for the tunneling of edge state excitations in fractional quantum Hall bars, the voltage bias leads only to different chemical potentials for the spatially separated left- and right-moving edges, see, e.g., P. Fendley, A.W.W. Ludwig, and H. Saleur, Phys. Rev. B 52, 8934 (1995). Our treatment applies to 1D interacting quantum wires.

[14] M. Abramowitz and I.A. Stegun, Handbook of Mathematical Functions (Dover, New York, 1971).

[15] Single Charge Tunneling, edited by H. Grabert and M.H. Devoret, NATO-ASI Series B: Vol.294 (Plenum 1992). 